\section{Island shapes and aggregation steered by the geometry of the substrate lattice $\dagger$}

\author{
M. B. Casu, ${ }^{* a}$ S.-A. Savu, ${ }^{a}$ B.-E. Schuster, ${ }^{a}$ I. Biswas, ${ }^{a}$ C. Raisch, ${ }^{a}$ H. Marchetto, ${ }^{b}$ \\ Th. Schmidt ${ }^{b}$ and T. Chassé ${ }^{a}$
}

Received 22nd February 2012, Accepted 16th May 2012

DOI: $10.1039 / \mathrm{c} 2 \mathrm{cc3} 3339 \mathrm{a}$

We find that island shapes and aggregation in diindenoperylene deposited on $\mathrm{Au}(100), \mathrm{Au}(110)$, and $\mathrm{Au}(111)$ single crystals are steered by the anisotropy due to the lattice geometry of the substrate. This phenomenon may be exploited as a tool for molecular patterning of surfaces.

Growth in the nanoscale regime has been described in detail since the 1990s with experiments and theories mainly focused on inorganic materials. ${ }^{1-4}$ Small organic molecules, colloids, and organic-based magnets are rapidly gaining attention towards future use in electronics. Their major and most significant difference with respect to systems described by classical theories is the growth unit, i.e., the building block is a molecule, not an atom. Introducing a new material in electronics demands methods to describe its growth onto a substrate, and the obtained interfaces in order to optimize the suitable properties for technology. This is one of the actual challenges in organic magnetism, ${ }^{5}$ while nucleation and kinetics pathways in materials like colloids seem to follow the same nucleation and growth laws that rule atomic growth. ${ }^{6}$

Here we use photoelectron emission microscopy (PEEM) and atomic force microscopy (AFM) to discuss how the island shape and aggregation in thin films of diindenoperylene (DIP, $\mathrm{C}_{32} \mathrm{H}_{16}$, Fig. 1) are influenced by the substrate lattice geometry.

DIP is a perylene-based molecule that shows a high hole mobility in thin films, good film forming properties, and thermal stability. ${ }^{7-9}$ It is a good candidate as a donor material in organic photovoltaic cells. ${ }^{10}$ The measurements were performed with the SMART (Spectro-Microscope with Aberration correction for many Relevant Techniques), ${ }^{11,12}$ an aberration-corrected spectromicroscope, installed at the UE49-PGM-b-SMART at BESSY (Berlin, Germany).

Fig. 1 shows an in situ observation of DIP growth on $\mathrm{Au}(111)$ by using UV-PEEM (Hg-lamp, $h \nu=4.9 \mathrm{eV})$. The PEEM images show bright and dark regions. The work function of gold is higher than the photon energy of the $\mathrm{Hg}$ lamp,

${ }^{a}$ Institute of Physical and Theoretical Chemistry,

University of Tübingen, Auf der Morgenstelle 18, D-72076 Tübingen, Germany.E-mail: benedetta.casu@uni-tuebingen

${ }^{b}$ Fritz Haber Institute of the Max Planck Society, Department of Chemical Physics, Faradayweg 4-6, D-14195 Berlin, Germany

$\dagger$ Electronic supplementary information (ESI) available. See DOI: $10.1039 / \mathrm{c} 2 \mathrm{cc} 31339 \mathrm{a}$
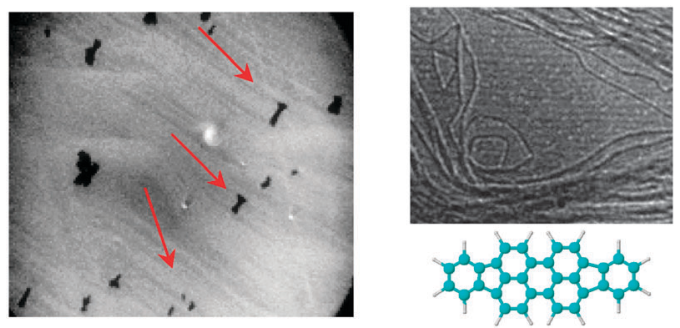

Fig. 1 UV-PEEM images of nominal $53 \AA$ thick DIP films showing islands on a $\mathrm{Au}(111)$ surface. The field of view is $25.0 \mu \mathrm{m} \times 18.7 \mu \mathrm{m}$. The arrows indicate anisotropic islands. The inset shows the herringbone reconstruction of the clean substrate (low energy electron microscopy (LEEM) image, the field of view is $2.0 \mu \mathrm{m} \times 1.5 \mu \mathrm{m}$ ). The DIP molecular structure $\left(\mathrm{C}_{32} \mathrm{H}_{16}\right)$ is also shown.

therefore there is no contribution from the substrate (no PEEM intensity is shown by the substrate). ${ }^{13}$ The work function decreases to $4.35 \mathrm{eV}$ within the first three DIP layers ${ }^{14}$ allowing the photoelectrons to escape yielding a bright PEEM image. With increasing film thickness, this contribution is attenuated by elastic and inelastic scattering of the photoelectrons that gives rise to dark regions. ${ }^{9,13}$ A layer-by-layer mechanism characterizes the initial growth (bright region) followed by island nucleation (dark regions), i.e., the growth follows the Stranski-Krastanov mode (layer plus islands). The islands show a clear branched shape. XPEEM investigations ${ }^{15}$ and thickness dependent measurements ${ }^{16}$ using X-ray photoemission spectroscopy further confirm this Stranski-Krastanov growth mode. DIP thin films on various substrates have been investigated, revealing the tendency to Stranski-Krastanov growth. ${ }^{8,9,14,17}$ In all cases, the observed islands were compact, i.e., without branches. In particular, we observed real time growth of DIP thin film on $\mathrm{Au}(100)$, under the same preparation conditions adopted in this work, obtaining compact islands. ${ }^{18}$

To check whether this particular PEEM result is not an artefact due to the electric field applied to the sample to accelerate or decelerate the electrons in order to obtain high lateral resolution and surface sensitivity, we performed an independent ex situ AFM experiment. We grew DIP thin films under exactly the same preparation conditions on $\mathrm{Au}(100), \mathrm{Au}(111)$, and $\mathrm{Au}(110)$ single crystals. During this experiment performed in a ultra high vacuum (UHV) chamber different from the PEEM, we used different single crystals (i.e., not the very same as in the PEEM experiments), 


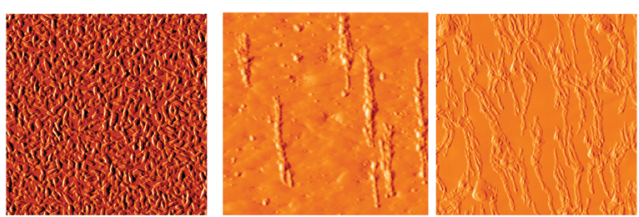

Fig. $210 \mu \mathrm{m} \times 10 \mu \mathrm{m}$ AFM images of DIP deposited on $\mathrm{Au}(100)$ (15 nm nominal thickness, left), Au(110) (16 nm nominal thickness, middle), and $\mathrm{Au}(111)$ (20 nm nominal thickness, right). The $z$-scale corresponds to the first derivative to better represent the island shape.

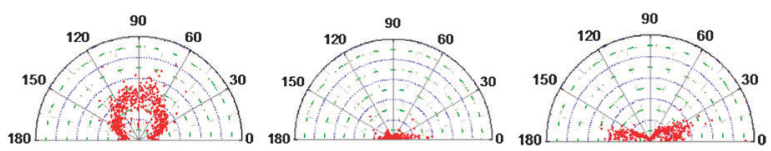

Fig. 3 Polar diagrams of the island angular distribution on $\mathrm{Au}(100)$ (left, $\theta=0^{\circ}$ is an arbitrary direction), $\mathrm{Au}(110)$ (middle, $\theta=0^{\circ}$ is the [1-10] in-plane direction), and $\mathrm{Au}(111)$ (right, $\theta=0^{\circ}$ is an arbitrary direction).

different evaporation geometry, the same Knudsen cell (i.e., exactly the same evaporation rate), and exactly the same preparation conditions as in the PEEM experiments. Fig. 2 shows the results. The difference in the morphology of films deposited on the different crystallographic faces is straightforward.

We have statistically analysed the AFM images (Fig. 3). The islands are compact and relatively small (the number of islands is correlated with island aggregation and size for samples of similar nominal film thickness) and no preferential orientation is shown on $\mathrm{Au}(100)$. They form aligned structures with the long axis along the $[1-10]$ in-plane direction on $\mathrm{Au}(110)$, and they are characterized by branches on $\mathrm{Au}(111)$ with a statistic angular distribution only along certain directions.

Island morphology depending on the substrate lattice geometry is not an isolated case with regard to DIP, although correlations have not been previously formulated as in the present work.

Recent works performed on 3,4,9,10-perylene-tetracarboxylicdianhydride (PTCDA) using scanning tunneling microscopy have been published by J. Ikonomov et al. ${ }^{19,20}$ on $\operatorname{Ag}(100)$, and by L. Kilian et al. ${ }^{21}$ on $\operatorname{Ag}(111)$. In both cases PTCDA is chemisorbed on silver. Sub-monolayers of PTCDA form islands that have a quadratic shape on $\mathrm{Ag}(100)$ at $120 \mathrm{~K}$, while sub-monolayers of PTCDA deposited on $\mathrm{Ag}(111)$ at $100 \mathrm{~K}$ are characterized by dendritic islands. Analogous effects have been explored for needles of para-hexaphenyl and sexithiophene grown on muscovite and phlogopite mica. ${ }^{22}$ The higher symmetry of phlogopite mica leads to a triangular structure in the arrangement of the needles, while on muscovite the needles grow in parallel. ${ }^{22,23} \mathrm{We}$ also performed a series of experiments showing that nanorods of terminally substituted pentacenes grown on $\mathrm{Au}(111)$ align according to the threefold geometry of the substrate. ${ }^{24}$

The lattice geometry of the substrate seems to be the common aspect influencing island shapes and aggregation in DIP, in PTCDA,${ }^{19-21}$ in para-hexaphenyl and sexithiophene, ${ }^{22,23}$ and in some substituted pentacenes, ${ }^{24}$ therefore, we intend to analyse the implications of different lattice geometries for interpretation of our results.

For fcc lattice substrates (see Fig. 4) the two low index surfaces, i.e., (100) and (110), are anisotropic, in particular the

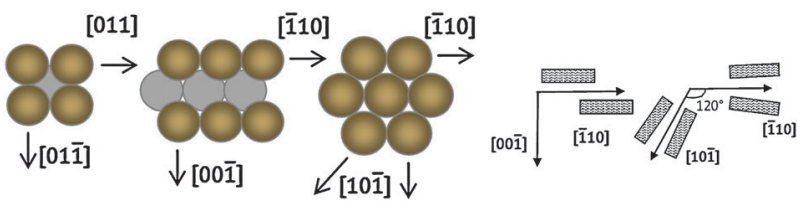

Fig. 4 Left panel: hard sphere schematic model of the fcc(100) (left), fcc(110) and fcc(111) surface planes. Right panel: a sketch of the island aggregation as inferred from AFM along the in-plane directions on $\mathrm{Au}(110)$ (left) and $\mathrm{Au}(111)$ (right) surfaces. Islands are displayed as rectangles.

reconstructed (100) surfaces show a large unit cell in which the interatomic distances are weakly anisotropic. The weak anisotropy in the case of the $\mathrm{Au}(100)$ substrate does not influence in a strong way the DIP island shape that is compact. The island distance is very small and the islands present similarities with DIP thin films grown on a variety of other substrates. ${ }^{8,9,14,17}$ In particular, the shape of the DIP islands on $\mathrm{Au}(100)$ has an evident similarity with those of DIP on polycrystalline gold, where the latter is rough and with no preferential surface lattice geometry, ${ }^{16}$ supporting the fact that the anisotropy in $\mathrm{Au}(100)$ is not sufficient to mark the island shape. The (110) faces show atomic troughs with strong anisotropy in the atomic distances giving rise to different degrees of possible interactions across or along the troughs. This anisotropy is the reason for the observed agglomeration of DIP islands elongated parallel to the [1-10] in-plane direction, similar to what is found in metal-on-metal epitaxy, for example, in $\mathrm{Cu}$ on $\operatorname{Pd}(110)^{3}$ a variety of elongated island structures can be obtained playing with temperature. $\mathrm{Au}(111)$ is a particular case because of the stress energy that is released via the herringbone reconstruction. For this reason the $\mathrm{Au}(111)$ surface is considered a patterned surface. ${ }^{3}$ The first DIP layer preserves the reconstruction ${ }^{15,16}$ and the islands grow assuming branched and fingered shapes. This anisotropy is due to the presence of two different possible types of steps on fcc(111) surfaces due to two structurally non-equivalent edges. ${ }^{3,4}$ In particular, steps running parallel to the [1-10] direction are found in $\mathrm{Au}(111)$, but also round and irregular steps along close-packed steps in low-index directions are observed. We may infer from the particular shape and island distribution in the PEEM and AFM images that DIP molecules are confined to follow only particular directions, because the diffusion may occur with different rates along these steps, leading to channels with preferential diffusion, as seen in transition from fractal to dendritic growth in metallic aggregation. ${ }^{25}$

At this point, it is important to identify and discuss the other parameters that may play a role in influencing the island shape, corroborating the above interpretation.

The first one is certainly temperature, ${ }^{1-4,26}$ since compact islands are expected for higher temperature and dendritic islands for lower temperature. ${ }^{4}$ The presence of islands showing branches only at their end (see the islands indicated by arrows in Fig. 1) could point to the fact that room temperature belongs to the "lower temperature range" for the gold + DIP system. Our experiment has been performed within a fixed temperature range, in order to minimize the number of parameters that influence the growth. ${ }^{26}$ In particular, all data shown here are obtained at $300 \pm 5 \mathrm{~K}$. Note that $\mathrm{Au}(100)$, $\mathrm{Au}(110)$ and $\mathrm{Au}(111)$ have different surface energies, ${ }^{1,3,27,28}$ 
i.e., different roughness increasing with surface energies, and different atom densities, ${ }^{1,3,27,28}$ (see Table 1 in ESI $\dagger$ ) thus we may infer that the molecular diffusion is facilitated on the $\mathrm{Au}(111)$ surface.

However, this observation has relevance to the formation of the first layer on top of the substrate. We observe StranskiKrastanov growth on each substrate with formation of a first layer on the substrate followed by island nucleation. Consequently, molecules landing on top of the first DIP layer should experience similar conditions, showing similar diffusion: we should observe similar island shapes on all surfaces, due to similar island-edge and Ehrlich-Schwoebel barriers ${ }^{4,16,29}$ that depend on thermal energy. ${ }^{30}$ The plausibility of a similar order of magnitude of the barriers across edges and corners is indirectly supported by the fact that in all three cases the film roughness has similar values (ranging from 30 to $40 \mathrm{~nm}$ as obtained by the AFM investigation) for the same thickness range. Consequently, we can rule out temperature as a main parameter influencing the island shape. Ehrlich-Schwoebel barrier depends also on orientation of the underlying molecules, torsion potential, and distance of the landing molecule from the step. ${ }^{30}$ We can assume that statistically the distance from the steps is similar in all cases, while the torsion potential is the same for the same molecule. Simbrunner et al. $^{22}$ and Clancy $^{30}$ have shown using appropriate calculations that a molecule prefers to adsorb with its long/short molecular axis at a certain angle on the substrate. This angle depends on the surface-molecule combination. ${ }^{22,30}$ DIP molecules form a layer of flat-lying molecules both on top of $\mathrm{Au}(111)$ and $\mathrm{Au}(100)$ single crystals. ${ }^{15,16,18}$ The role of the substrate is still relevant also for the layers after the first one, influencing the specific orientation of the molecules, leading to molecules adopting a recumbent position on all three substrates. ${ }^{15,16,18,31}$ Changes in molecular orientation with thickness reflect a different equilibrium between moleculemolecule and molecule-substrate interactions. In pentacene, the morphology of the thin films is shown to be a function of the molecule-molecule and molecule-substrate interactions. ${ }^{30}$ Given the same preparation, DIP physisorption on gold, the similar recumbent arrangement, the order of magnitude of the strength of these two interactions is expected to be similar for DIP on all three different gold single crystals. Thus, at this point, we can also rule out molecular orientation, intermolecular and molecule-substrate interactions as parameters causing the difference in island shape.

In conclusion, we find that when depositing thin films of DIP on $\mathrm{Au}(111), \mathrm{Au}(100)$, and $\mathrm{Au}(110)$ single crystals, the island shape and aggregation are steered by the anisotropy due to the substrate lattice geometry. The role of the first layer in DIP is still an open question, since we observe the steering of the island shape by the lattice geometry in the Stranski-Krastanov growth mode, contrary to the fact that this effect was previously found in the Volmer-Weber growth mode (i.e., pure island nucleation) of metals and other organic molecules.

A quantitative description of the phenomena reported here needs undoubtedly realistic simulations. We think that our work elucidates further aspects influencing organic thin film growth and may stimulate the necessary theoretical modelling and calculations. In addition, the different degrees of anisotropy in different lattice geometries may be exploited in the future as a tool for molecular patterning of specific surfaces.

The authors thank BESSY, in particular Dr W. Braun, for providing beamtime, Prof. H. Freund and Prof. E. Umbach for the SMART project, W. Neu for technical support. Financial support by the BMBF is gratefully acknowledged.

\section{Notes and references}

1 J. A. Venables, Introduction to Surface and Thin Film Processes, Cambridge University Press, Cambridge, 2000.

2 E. Bauer, Z. Kristallogr., 1958, 110, 372.

3 H. Brune, Surf. Sci. Rep., 1998, 31, 121.

4 Z. Zhang and M. G. Lagally, Science, 1997, 276, 377.

5 D. Gatteschi, A. Cornia, M. Mannini and R. Sessoli, Inorg. Chem., 2009, 48, 3408.

6 R. Ganapathy, M. R. Buckley, S. J. Gerbode and I. Cohen, Science, 2010, 327, 445.

7 N. Karl, Synth. Met., 2003, 133-134, 649.

8 S. Kowarik, A. Gerlach, S. Sellner, F. Schreiber, L. Cavalcanti and O. Konovalov, Phys. Rev. Lett., 2006, 96, 125504.

9 M. B. Casu, I. Biswas, M. Nagel, P. Nagel, S. Schuppler and T. Chassé, Phys. Rev. B, 2008, 78, 075310.

10 J. Wagner, M. Gruber, A. Hinderhofer, A. Wilke, B. Bröker, J. Frisch, P. Amsalem, A. Vollmer, A. Opitz, N. Koch, F. Schreiber and W. Brütting, Adv. Funct. Mater., 2010, 20, 4295.

11 R. Wichtendahl, R. Fink, H. Kuhlenbeck, D. Preikszas, H. Rose, R. Spehr, P. Hartel, W. Engel, R. Schlögl, H. J. Freund, A. M. Bradshaw, G. Lilienkamp, Th. Schmidt, E. Bauer, G. Benner and E. Umbach, Surf. Rev. Lett., 1994, 5, 1249.

12 R. Fink, M. R. Weiss, E. Umbach, D. Preikszas, H. Rose, R. Spehr, P. Hartel, W. Engel, R. Degenhardt, R. Wichtendahl, H. Kuhlenbeck, W. Erlebach, K. Ihmann, R. Schlögl, R. H. J. Freund, A. M. Bradshaw, G. Lilienkamp, Th. Schmidt, E. Bauer and G. Benner, J. Electron Spectrosc. Relat. Phenom., 1997, 84, 231.

13 H. Marchetto, Doctoral Thesis, Freie Universität, Berlin, 2006.

14 A. C Dürr, N. Koch, M. Kelsch, A. Rühm, J. Ghijsen, R. L. Johnson, J.-J. Pireaux, J. Schwartz, F. Schreiber, H. Dosch and A. Kahn, Phys. Rev. B, 2003, 68, 115428.

15 M. B. Casu, B.-E. Schuster, I. Biswas, C. Raisch, H. Marchetto, Th. Schmidt and T. Chassé, Adv. Mater., 2010, 22, 3740.

16 M. B. Casu, Cryst. Growth Des., 2011, 11, 3629.

17 D. G. de Oteyza, T. N. Krauss, E. Barrena, S. Sellner, H. Dosch and J. S. Ossó, Appl. Phys. Lett., 2007, 90, 243104.

18 M. B. Casu, S.-A. Savu, P. Hoffmann, B.-E. Schuster, O. Mentes, M. A. Niño, A. Locatelli and T. Chassé, CrystEngComm, 2011, 13, 4139.

19 J. Ikonomov, O. Bauer and M. Sokolowski, Surf. Sci., 2008, 602, 2061.

20 J. Ikonomov, C. H. Schmitz and M. Sokolowski, Phys. Rev B, $2010, \mathbf{8 1}, 195428$.

21 L. Kilian, A. Hauschild, R. Temirov, S. Soubatch, A. Schöll, A. Bendounan, F. Reinert, T.-L. Lee, F. S. Tautz, M. Sokolowski and E. Umbach, Phys. Rev. Lett., 2008, 100, 136103.

22 C. Simbrunner, D. Nabok, G. Hernandez-Sosa, M. Oehzelt, T. Djuric, R. Resel, L. Romaner, P. Puschnig, C. Ambrosch-Draxl, I. Salzmann, G. Schwabegger, I. Watzinger and H. Sitter, J. Am. Chem. Soc., 2011, 133, 3056.

23 F. Balzer, M. Schiek, H.-G. Rubahn, K. Al-Shamery and A. Lutzen, J. Vac. Sci. Technol., B, 2008, 26, 1619.

24 S.-A. Savu, M. B. Casu, S. Schundelmeier, S. Abb, C. Tönshoff, H. F. Bettinger and Th. Chassé, RSC Adv., 2012, 2, 5112.

25 H. Brune, C. Romainczyk, H. Röder and K. Kern, Nature, 1994, 369, 469 .

26 M. B. Casu, A. Schöll, K. R. Bauchspieß, D. Hübner, Th. Schmidt, C. Heske and E. Umbach, J. Phys. Chem. C, 2009, 113, 10990.

27 K. W. Kolasinski, Surface Science, Wiley, England, 2008.

28 L. Vitos, A. V. Ruban, H. L. Skriver and J. Kollár, Surf. Sci., 1998, 411, 186.

29 G. Ehrlich and F. G. Hudda, J. Chem. Phys., 1966, 44, 1039; R. L. Schwoebel and E. J. Shipsey, J. Appl. Phys., 1966, 37, 3682.

30 P. Clancy, Chem. Mater., 2011, 23, 522.

31 We measured near edge X-ray absorption fine structure spectroscopy on DIP deposited on $\mathrm{Au}(110)$ obtaining evidence for a recumbent position (unpublished result). 